\title{
Clinical utility gene card for: Alström Syndrome - update 2013
}

\author{
Jan D Marshall ${ }^{\star, 1}$, Pietro Maffei ${ }^{2}$, Sebastian Beck ${ }^{3}$, Timothy G Barrett ${ }^{4}$, Richard Paisey ${ }^{5}$ and \\ Jürgen K Naggert ${ }^{1}$
}

European Journal of Human Genetics (2013) 21, doi:10.1038/ejhg.2013.61; published online 24 April 2013

Update to: European Journal of Human Genetics (2011) 19, 1108; doi:10.1038/ejhg.2011.72; published online 27 April 2011

\section{DISEASE CHARACTERISTICS}

1.1 Name of the disease (synonyms)

Alström syndrome, ALMS.

\subsection{OMIM\# of the disease}

203800.

1.3 Name of the analysed genes or DNA/chromosome segments ALMS1.

\subsection{OMIM\# of the gene(s)}

606844.

\subsection{Mutational spectrum}

There have been 106 different disease-causing mutations described thus far, including nonsense (55\%), indels (42\%), and rare compound frameshift, and splice site (3\%) mutations. ${ }^{1-6}$ Several missense alterations of uncertain pathogenicity have been reported. ${ }^{7}$ The majority of all mutations are clustered in exon 16 (41\%), exon 10 (27\%) and exon 8 (25\%) of ALMS1. ${ }^{2}$ Compound heterozygosity is common. Chromosomal translocations with a breakpoint through ALMS1, an insertion of AluYa5 elements, intronic mutations, and large deletions are rare but have also been reported. ${ }^{7-9}$

\subsection{Analytical methods}

Bi-directional sequencing of coding exons of ALMS1, including their flanking intronic sequences. If the family structure is appropriate, linkage analysis for ALMS1 may be useful to pre-screen, owing to the large size of the gene. Conventional cytogenetics is usually normal.

\subsection{Analytical validation}

Both strands are sequenced. When a mutation is identified, validation of the results using a second primer set or a second technique (PCR with restriction enzyme digestion) is recommended, as well as external validation for DNA sequencing through international quality assurance schemes, when possible. Segregation analysis by sequencing the DNA of the parents is usually necessary to confirm the pathogenic status of two mutations found in patients. Interpretation of the molecular genetic results can be ambiguous, as synonymous substitutions, SNPs and missense mutations can be non-disease causing (neutral).
1.8 Estimated frequency of the disease (incidence at birth ('birth prevalence') or population prevalence)

The population prevalence is unknown, but is thought to range between 1:500 000 and 1:1000 $000 .^{2}$

1.9 If applicable, prevalence in the ethnic group of investigated person

There is a higher frequency of some particular ALMS1 mutations in certain ethnic populations, for example, 10535ins(n)19 in French Acadians, and $10775 \mathrm{delC}$ in up to $20 \%$ of patients of English descent. ${ }^{1,10}$ As seen in many autosomal recessive diseases, the prevalence is higher in isolated populations or where consanguineous marriages are common. ${ }^{11}$

\subsection{Diagnostic setting}

\begin{tabular}{lll}
\hline & Yes & No \\
A. (Differential) diagnostics & $\bigotimes$ & $\square$ \\
B. Predictive testing & $\Downarrow$ & $\square$ \\
C. Risk assessment in relatives & $\Downarrow$ & $\square$ \\
D. Prenatal & $\Downarrow$ & $\square$
\end{tabular}

\section{Comment:}

Several other conditions have overlapping phenotypes, and should be evaluated in the differential diagnosis, particularly in the young child: achromatopsia, Leber congenital amaurosis (LCA), idiopathic cardiomyopathy in infants, and Bardet-Biedl syndrome (BBS). BBS is predominately characterized by obesity, polydactyly, developmental delay and learning difficulties, and rod-cone dystrophy, with a later onset and slower progression with no photophobia. Hearing loss is less common in BBS. A first diagnosis of LCA is often considered in the young infant without dilated cardiomyopathy, but should be reconsidered if additional symptoms develop. In an infant with nystagmus, photophobia, and cardiomyopathy, Alström syndrome should be considered first. Conversely, idiopathic cardiomyopathy in infants who also have cone-rod retinal degeneration is a strong indicator of Alström syndrome. Other disorders sometimes included in the differential diagnosis that could lead to mis-diagnosis are: mitochondrial dysfunction, retinitis pigmentosa with hearing loss, Usher syndrome, and Wolfram syndrome. ${ }^{12}$

Prenatal and predictive diagnosis can be undertaken if a mutated allele is identified in each of the parents. Predictive diagnosis is only of

\footnotetext{
${ }^{1}$ The Jackson Laboratory, Bar Harbor, ME, USA; ${ }^{2}$ Department of Medicine, Padua University Hospital, Padua, Italy; ${ }^{3}$ Praxis Blaues Haus, Kreuzlingen, Switzerland; ${ }^{4}$ School of Clinical and Experimental Medicine, University of Birmingham, Birmingham, UK; ${ }^{5}$ Torbay Hospital, Lawes Bridge, Torquay, UK

*Correspondence: JD Marshall, The Jackson Laboratory, 600 Main Street, Bar Harbor, ME 04609 , USA. Tel: + 12072886385 ; Fax: +1 207 288 6078 ;

E-mail: jan.marshall@jax.org
} 
limited relevance because the first symptoms of Alström syndrome appear in infancy.

\section{TEST CHARACTERISTICS}

\begin{tabular}{|c|c|c|c|c|}
\hline & \multicolumn{2}{|c|}{ Genotype or disease } & \multirow{2}{*}{$\begin{array}{l}\text { A: true positives } \\
\text { B: false positives }\end{array}$} & \multirow{2}{*}{$\begin{array}{l}\text { C: false negatives } \\
\text { D: true negatives }\end{array}$} \\
\hline & Present & Absent & & \\
\hline \multicolumn{5}{|l|}{ Test } \\
\hline \multirow[t]{2}{*}{ Positive } & A & $B$ & Sensitivity: & $A /(A+C)$ \\
\hline & & & Specificity: & $D /(D+B)$ \\
\hline \multirow[t]{2}{*}{ Negative } & C & $\mathrm{D}$ & Positive predictive value: & $A /(A+B)$ \\
\hline & & & Negative predictive value: & $D /(C+D)$ \\
\hline
\end{tabular}

\subsection{Analytical sensitivity}

(proportion of positive tests if the genotype is present)

Depending upon the method used, the proportion of both mutations found in this recessive disease is about $85 \%$; in $<15 \%$ only one of the two mutations will be found. In about $0.6 \%$ neither of the mutations will be found with the method described (DNA sequencing only). Only one mutation found in the context of appropriate clinical presentation may confirm diagnosis of Alström syndrome. ${ }^{12}$ A negative result using current methods does not exclude the diagnosis if classic clinical features are present. The gene is highly polymorphic and we currently lack a functional assay to assess the pathogenicity of some variants.

\subsection{Analytical specificity}

\section{(proportion of negative tests if the genotype is not present)}

Nearly $100 \%$. In some cases, some variants may erroneously be interpreted as pathogenic.

\subsection{Clinical sensitivity}

\section{(proportion of positive tests if the disease is present)}

The clinical sensitivity can be dependent on variable factors such as age or family history. In such cases a general statement should be given, even if a quantification can only be made case by case.

The clinical sensitivity depends upon the clinical criteria used. In over $95 \%$ of the patients who fulfill the criteria of Marshall et al $l^{12}$ at least one mutation is found. The phenotype can be quite variable. Given the rarity of the syndrome and the progressive phenotype, depending on the age of the patient and the clinical symptoms, it can be concluded that a finding of only one mutation together with age-appropriate symptoms may confirm or 'strongly suggest' the diagnosis of Alström syndrome. ${ }^{12}$

\subsection{Clinical specificity}

(proportion of negative tests if the disease is not present)

The clinical specificity can be dependent on variable factors such as age or family history. In such cases a general statement should be given, even if a quantification can only be made case by case.

The clinical specificity is estimated to be nearly $100 \%$. However, it should be taken into account that the mild end of the phenotypic spectrum is not yet completely known, so some individuals with Alström syndrome may be missed.

\subsection{Positive clinical predictive value}

(life-time risk to develop the disease if the test is positive)

Alström syndrome develops in infancy. If ALMS1 mutations are identified in the child, the risk of developing further symptoms of the disorder is $100 \%$. However, there is considerable variability and some phenotypes are more predictable than others. There have been no reported cases of incomplete penetrance.

\subsection{Negative clinical predictive value}

(probability not to develop the disease if the test is negative)

Assume an increased risk based on family history for a non-affected person. Allelic and locus heterogeneity may need to be considered.

Index case in that family had been tested:

When a pathogenic ALMS1 mutation is identified in the index case, the negative predictive value is $100 \%$.

Index case in that family had not been tested:

Normally predictive tests are not performed in such cases. Further testing for family members may be appropriate in rare circumstances, but should only be proposed when both of the pathogenic mutations have been identified in an index case.

\section{CLINICAL UTILITY}

\section{1 (Differential) diagnostics: The tested person is clinically} affected

(To be answered if in 1.10 'A' was marked)

\subsubsection{Can a diagnosis be made other than through a genetic test?}

\begin{tabular}{lll}
\hline No & $\square$ (continue with 3.1.4) & \\
Yes & $\bigotimes$ & \\
& Clinically & \\
& Imaging & $\square$ \\
& Endoscopy & $\square$ \\
& Biochemistry & $\square$ \\
& Electrophysiology & $\square$ \\
\hline
\end{tabular}

Comment:

The clinical diagnosis of Alström syndrome in an infant or very young child requires the presence of a characteristic global retinal dystrophy with major initial cone involvement in childhood, with photophobia AND either obesity or cardiomyopathy. Confirmation of the nature of the retinal dystrophy by ERG might be very helpful in the early diagnostic process. Some cases present with later-onset vision loss (1-3 years), and some have been non-obese. In childhood, early adolescence, and adulthood, additional phenotypes evolve. Insulin-resistant diabetes can occur from childhood and may be preceded by acanthosis nigricans. Triglycerides are often elevated. Many patients develop cardiomyopathy in infancy or adolescence. Recurrent lower respiratory tract infections are common in early childhood, and non-alcoholic fatty liver disease can develop in early childhood or during puberty. Chronic renal failure is seen with variable severity in up to $84 \%$ of patients during the second decade. Multiple organ fibrosis is found at post mortem.

In Alström syndrome, the combination of the late onset of some features, such as cardiac, hepatic, and renal disease, and the existence of other genetic syndromes with similar cardinal manifestations, such as BBS, often leads to confusion among clinicians and possible misdiagnosis. Alström syndrome belongs to a growing class of human diseases, referred to as ciliopathies.

The diagnosis of Alström syndrome is often difficult in young children because many of the clinical features (hearing loss, insulin resistance, diabetes, and congestive heart failure) develop over time. Therefore, diagnostic requirements differ as the child grows, underscoring the challenge in such cases. To address phenotypic variability and the progressive nature of these clinical features, a three-tiered set 
of diagnostic criteria that takes into account the patient's age has been recommended for correct diagnosis of Alström syndrome. ${ }^{12}$

\subsubsection{Describe the burden of alternative diagnostic methods to the patient}

Diagnosis can be established on the basis of a constellation of appropriate clinical features, and laboratory and instrumental assessments (vision and hearing loss, retinal dystrophy with early cone involvement by electroretinogram, echocardiography, presence of metabolic abnormalities), depending upon the age of the patient. ${ }^{12}$

3.1.3 How is the cost effectiveness of alternative diagnostic methods to be judged?

Unknown. Genetic testing is still expensive and the unavailability of affordable genetic testing or unusual clinical criteria can cause some individuals with Alström syndrome to be missed. However, the emergence of new genetic tools such as next-generation sequencing will allow for genetic confirmation at a reasonable price for more individuals. Genetic testing may be particularly important in patients who do not have all of the classical Alström characteristics and may be at risk for the long-term complications of the syndrome.

\subsubsection{Will disease management be influenced by the result of a genetic test?}

No. $\square$

Yes. $\square$

Therapy (please describe)

Prognosis (please describe)

Management (please describe)

Treatment is based upon symptoms and indications for drug therapy (type 2 diabetes, hypertension, cardiac function, hormonal imbalances), and are not altered in patients with a molecular diagnosis as compared to those in whom an ALMS1 mutation has not been identified. Appropriate treatment should be prescribed for all patients with a diagnosis of Alström syndrome based upon clinical features and investigations alone. Despite optimal clinical care, the prognosis is poor in many patients. The identification of an ALMS1 mutation will not lead to a different prognosis when compared with patients with Alström syndrome for whom mutations have not been identified.

Clinical management is complex. Regular assessments for vision and hearing loss, as well as echocardiography follow-up and monitoring of metabolic alterations should occur with or without identification of the ALMS1 molecular defect. Blindness and hearing loss should be anticipated, so special adaptations should be made available in the educational setting. When possible, patients should be integrated in a multi-disciplinary clinic to anticipate and screen for treatable comorbidities including hypertension, non-alcoholic fatty liver disease, glucose abnormalities, microalbuminuria and lipid abnormalities. The results of genetic testing will influence genetic counselling and diagnosis of younger affected children in the same family. Patients without a molecular diagnosis might be more frequently subjected to diagnostic myocardial or liver biopsy procedures and multiple specialized assessments in comparison to patients with known mutations.

3.2 Predictive setting: The tested person is clinically unaffected but carries an increased risk based on family history

(To be answered if in 1.10 ' $\mathrm{B}$ ' was marked)
3.2.1 Will the result of a genetic test influence lifestyle and prevention? To our knowledge, predictive testing has not been carried out yet, but preimplantation and prenatal testing have. However, it might be useful to perform predictive testing in a yet unaffected neonate, sibling to a patient with Alström syndrome with two known mutations. First symptoms occur during the first months of life and the description of the consequences applies for healthy neonates with an increased risk based on family history.

If the test result is positive (please describe):

In early childhood there is a high risk to develop acute dilated cardiomyopathy, often misdiagnosed as idiopathic and sometimes with fatal outcome. Therefore monitoring of heart function should be mandatory, as well as teaching the parents in early recognition of the symptoms of heart failure that are not easy to detect in the early months of life.

The widespread major organ fibrosis can be occult, resulting in underestimation of the risk of cardiorespiratory death in the context of pneumonia or anaesthesia. Early intensive care assessment is vital in these circumstances.

If the test result is negative (please describe):

In some affected patients, ALMS1 mutations may not be identified. A negative genetic test in the context of the appropriate clinical phenotype does not exclude the diagnosis of Alström syndrome and the described precautions are recommended throughout life. However, in the case of a negative test result and the absence of clinical symptoms, no additional testing is needed unless the neonate presents with symptoms.

\subsubsection{Which options in view of lifestyle and prevention does a person} at-risk have if no genetic test has been done (please describe)? Regular clinical monitoring, particularly heart, liver, growth, endocrine and metabolic parameters, and renal function if vision loss begins or if other symptoms develop.

3.3 Genetic risk assessment in family members of a diseased person (To be answered if in 1.10 ' $\mathrm{C}$ ' was marked)

\subsubsection{Does the result of a genetic test resolve the genetic situation in that family?}

Yes-autosomal recessive inheritance.

3.3.2 Can a genetic test in the index patient save genetic or other tests in family members?

Yes, family members who are possible carriers can be screened for the causative ALMS1 mutations if both mutated alleles have been identified in the index case.

3.3.3 Does a positive genetic test result in the index patient enable a predictive test in a family member?

Yes, but there is very early disease onset in most cases, so predictive testing is normally not necessary. However, it may be possible to test a younger sibling of an index patient before the development of the first symptoms.

\subsection{Prenatal diagnosis}

(To be answered if in 1.10 ' $\mathrm{D}$ ' was marked)

\subsubsection{Does a positive genetic test result in the index patient enable a} prenatal diagnosis?

Yes, preimplantation and prenatal diagnosis can be undertaken if both mutated ALMS1 alleles are identified in the parents. 


\section{IF APPLICABLE, FURTHER CONSEQUENCES OF TESTING}

Please assume that the result of a genetic test has no immediate medical consequences. Is there any evidence that a genetic test is nevertheless useful for the patient or his/her relatives? (Please describe).

The diagnosis has huge consequences for patients and relatives. Apart from medical consequences, educational and social intervention for a child is essential. Therefore, an unambiguous diagnosis is of great value. Additionally, providing 'an answer' for patients and parents allows them to seek contact with other persons with Alström syndrome or with patient organizations, which can be of great benefit in coping with the condition.

There is little benefit to clarify the carrier status in a relative of a patient with Alström syndrome, as long as his/her spouse is not also a relative of a patient with Alström. In case the patient wishes to know his carrier status, he should receive qualified genetic counseling.

\section{CONFLICT OF INTEREST}

The authors declare no conflict of interest.

\section{ACKNOWLEDGEMENTS}

This work was supported by EuroGentest, an EU-FP6-supported NoE, contract number 512148 (EuroGentest Unit 3: 'Clinical genetics, community genetics and public health', Workpackage 3.2). JDM and JKN were supported by a National Institutes of Health grant HD036878. PM and TGB were funded by Executive Agency for Health and Consumer (EAHC), EURO-WABB, agreement number 201012 05. TGB and RP were funded by the UK National Specialist Clinical Team, NSCT.

1 Marshall JD, Hinman EG, Collin GB et al: Spectrum of ALMS1 variants and evaluation of genotype-phenotype correlations in Alström syndrome. Hum Mutation 2007; 28 1114-1123.

2 Marshall JD, Maffei P, Collin GB, Naggert JK: Alström syndrome: genetics and clinical overview. Curr Genomics 2011; 12: 225-235.

3 Pereiro I, Hoskins BE, Marshall JD et al: Arrayed Primer Extension (APEX) technology simplifies mutation detection in Bardet Biedl and Alström Syndrome. Eur J Hum Genet 2011; 19: 485-488.

4 Piñeiro-Gallego T, Cortón M, Ayuso C, Baiget M, Valverde D: Molecular approach in the study of Alström syndrome: analysis of ten Spanish families. Mol Vis 2012; 18: 1794-1802.

5 Redin C, Le Gras S, Mhamdi 0 et al: Targeted high-throughput sequencing for diagnosis of genetically heterogeneous diseases: efficient mutation detection in Bardet-Biedl and Alström Syndromes. J Med Genet 2012; 49: 502-512.

6 LOVD Open Access Online Mutation Database. www.euro-wabb.org

7 Joy T, Cao H, Black G et al: Alström syndrome (OMIM 203800): a case report and literature review. Orphanet J Rare Dis 2002; 1: 49.

8 Hearn T, Renforth GL, Spalluto $C$ et al: Mutation of $A L M S 1$, a large gene with a tandem repeat encoding 47 amino acids, causes Alström syndrome. Nat Genet 2002; 31 79-83.

9 Tașkesen M, Collin GB, Evsikov AV, Güzel A, Ozgül RK, Marshall JD et al: Novel Alu retrotransposon insertion leading to Alström syndrome. Hum Genet 2012; 13: 407-413.

10 Collin GB, Marshall JD, Ikeda A et al: Mutations in ALMS1 cause obesity, type 2 diabetes and neurosensory degeneration in Alström syndrome. Nat Genet 2002; 31 : 74-78.

11 Aldahmesh MA, Abu-Safieh L, Khan AO et al: Allelic heterogeneity in inbred populations: the Saudi experience with Alström syndrome as an illustrative example. Am J Med Genet A 2009; 149A: 662-665.

12 Marshall JD, Beck S, Maffei P, Naggert JK: Alström syndrome. Eur J Hum Genet 2007, 15: 1193-1202. 